\title{
Rosai-Dorfman Disease of the Spine Masquerading as Meningioma
}

\author{
Vijay P. Joshi ${ }^{1}$ Prasanna Kasegaonkar ${ }^{2}$ Gauri Kelkar ${ }^{3}$ Seena Kasegaonkar ${ }^{4}$ Mrugakshi Khairnar \\ Amit Agrawal ${ }^{5}$
}

${ }^{1}$ Department of Neurosurgery, Chandan Neurosciences Hospital, Solapur, Maharashtra, India

${ }^{2}$ Department of Neurology, Chandan Neurosciences Hospital, Solapur, Maharashtra, India

${ }^{3}$ Department of Pathology, Chandan Neurosciences Hospital, Solapur, Maharashtra, India

${ }^{4}$ Department of Radiology, Chandan Neurosciences Hospital, Solapur, Maharashtra, India

${ }^{5}$ Department of Neurosurgery, Narayana Medical College Hospital, Chinthareddypalem, Nellore, Andhra Pradesh, India

Indian ] Neurosurg 2019;8:86-88

\begin{abstract}
Address for correspondence Dr. Vijay P. Joshi, MCh, Consultant Neurosurgeon, Chandan Neurosciences Hospital, Solapur, Maharashtra, India (e-mail: 1974vpjjoshi@gmail.com).
\end{abstract}

S100 protein and CD30 did not reveal any R-S cells. Special stains for microorganisms were negative. Postoperatively the patient gradually improved in her paraparesis and sensory symptoms. On discharge, she had grade $4 / 5$ power in all muscle groups of the lower limbs. She is doing well at 1-year follow-up.

The exact etiology of RDD is not known and it is considered a benign idiopathic histiocytosis. Infectious agents (i.e., human herpes virus 6 [HHV-6] and parvovirus B19) or an abnormal immunologic response have been suggested as causative factors. ${ }^{4,8}$ Although there is no age preponderance for the CNS involvement in RDD, the disease usually involves male patients between 20 and 40 years of age. ${ }^{1,2}$ The systemic RDD most commonly involves the orbit, skin, respiratory tract, bones, kidneys, heart, and head and neck region, and is characterized by massive and painless cervical lymphadenopathy with variable symptoms of fever, leukocytosis, raised erythrocyte sedimentation rate (ESR), and weight loss..$^{5,910}$ Involvement of the spine is uncommon, and usually there is absence of lymphadenopathy. ${ }^{11}$

It is difficult to make a diagnosis of isolated RDD on imaging as MRI features of this disease are almost similar to other common lesions of the spinal cord (i.e., meningiomas). ${ }^{4}$ The signal intensity of these lesions on MRI ranges from iso- to hypointensity on T1W images to iso- to hyperintensity on T2W images, and after the contrast administration, the lesions show an intense and uniform enhancement (including dural tail). ${ }^{12,13}$ Because of this, the definitive diagnoses are possible only after the histopathology (if necessary immunohistochemistry), which will be the characteristic pattern of the disease. ${ }^{4,12}$ On histopathology, RDD is characterized by emperipolesis "phagocytosis of intact lymphocytes by macrophages" and received

November 13, 2016

accepted after revision

April 30, 2018

published online

April 23, 2019
DOI https://doi.org/

10.1055/s-0039-1677965

ISSN 2277-954X.
¿2019 Neurological Surgeons'

Society of India
License terms

()(1) $\Theta \circledast$ 


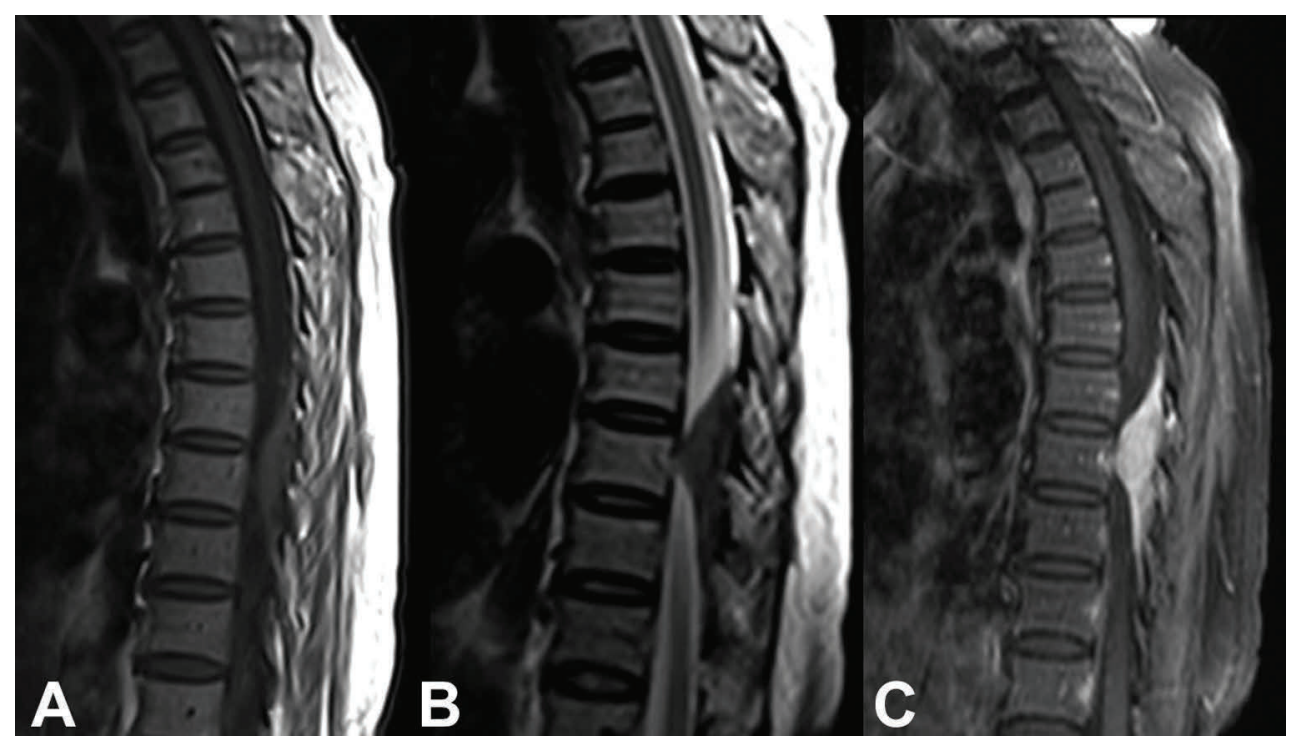

Fig. 1 MRI T1W (A), T2W (B), and axial images showing extradural hypointense lesion compressing the cord and pushing it toward left side. MRI dorsal spine postcontrast sagittal image (C) showing intensely enhancing lesion with dural tail.

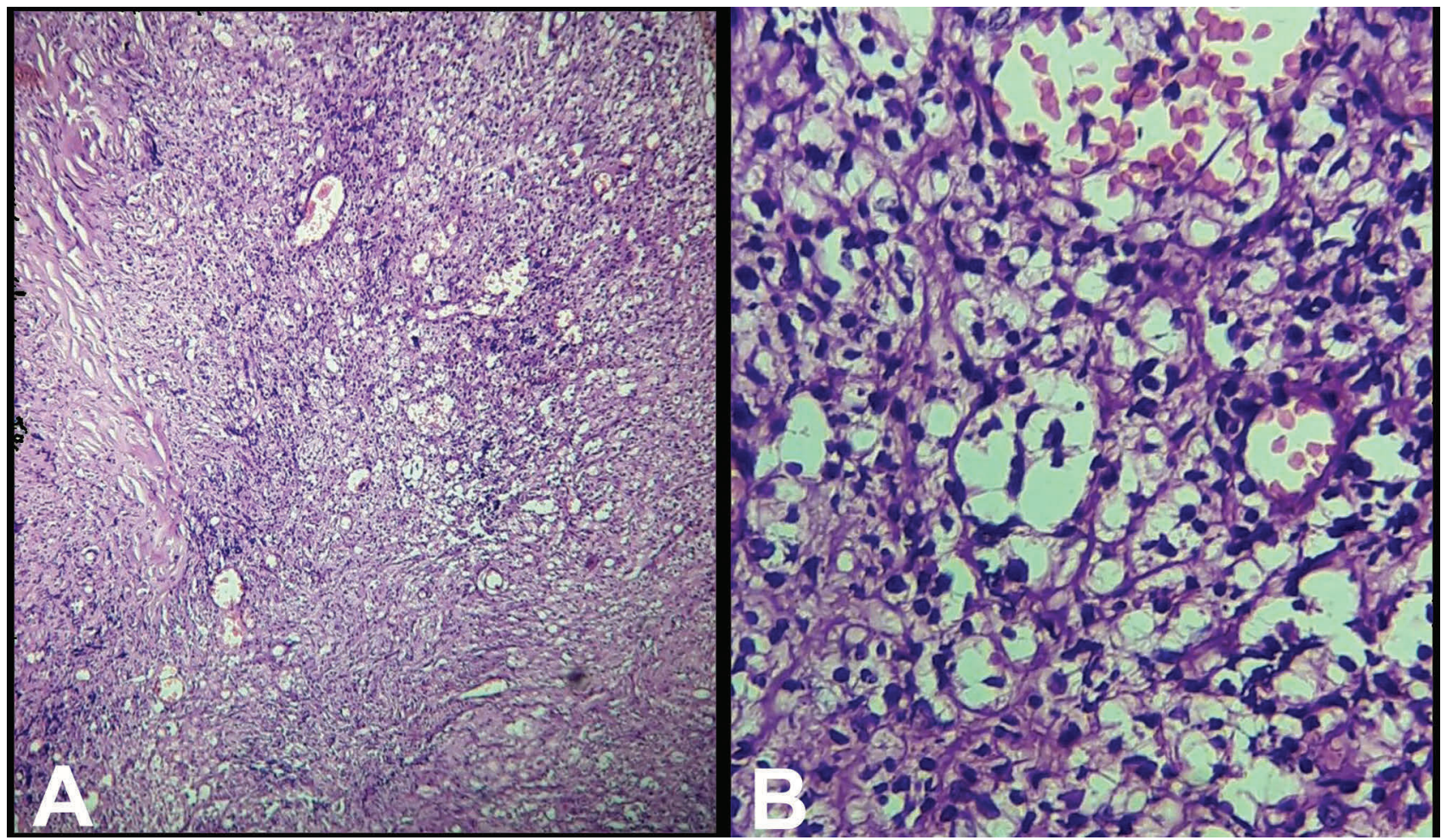

Fig. 2 (A) $\mathrm{H}$ and $\mathrm{E}$ stain 10X magnification-microscopic examination demonstrating that mixed inflammatory background consists of lymphocytes, plasma cells, and polymorphonuclear leukocytes. Singly scattered and clusters of pale-staining histiocytes seen and (B) $\mathrm{H}$ and $\mathrm{E}$ stain 40X magnification-microscopic examination demonstrating that mixed inflammatory background consists of lymphocytes, plasma cells, and polymorphonuclear leukocytes. Singly scattered and clusters of pale-staining histiocytes demonstrating that emperipolesis are seen. Foci of fibrosis are noted.

presence of large histiocytes immunoreactive for S100 protein and CD68. ${ }^{14,14}$ These cells are negative for CD1 distinguishing from Langerhans' cell histiocytosis. ${ }^{15}$

The mainstay of treatment is surgical decompression, and the main objective of the management of spinal RDD is to relieve the compression over the neural structures., ${ }^{7,16-18}$ In cases of recurrent or progressive cases of RDD, several therapies (e.g., radiotherapy and combination of chemotherapy with corticosteroids, 6-mercaptopurine, and methotrexate) with variable success have been reported. ${ }^{13,19}$ The natural history of RDD ranges from complete and spontaneous remission, periodic exacerbations and remissions, and persistence of the disease, and, in some cases, it may be associated with fatal outcome due to systemic complications. ${ }^{10,13}$ 
Isolated involvement of the spine in RDD is uncommon, and it needs to be considered in the differential diagnosis of patients presenting with spinal cord compression. Early surgical intervention, resection of the lesion, and relief in the mass effect help increase chances of recovery of neurologic functions.

\section{References}

1 Rosai J, Dorfman RF. Sinus histiocytosis with massive lymphadenopathy. A newly recognized benign clinicopathological entity. Arch Pathol 1969;87(1):63-70

2 Hollowell JP, Wolfla CE, Shah NC, Mark LP, Whittaker MH. Rosai-Dorman disease causing cervical myelopathy. Spine 2000;25(11):1453-1456

3 Al-Saad K, Thorner P, Ngan B-Y, et al. Extranodal Rosai-Dorfman disease with multifocal bone and epidural involvement causing recurrent spinal cord compression. Pediatr Dev Pathol 2005;8(5):593-598

4 Bhandari A, Patel PR, Patel MP. Extranodal Rosai-Dorfman disease with multiple spinal lesions: a rare presentation. Surg Neurol 2006;65(3):308-311

5 Maiti TK, Gangadharan J, Mahadevan A, Arivazhagan A, Chandramouli BA, Shankar SK. Rosai-Dorfman disease presenting as cervical extradural lesion: a case report with review of literature. Neurol India 2011;59(3):438-442

6 Osenbach RK. Isolated extranodal sinus histiocytosis presenting as an intramedullary spinal cord tumor with paraplegia. Case report. J Neurosurg 1996;85(4):692-696

7 Xin Y, Shen H, Kong D, Jia W, Liu H, Yang J. Isolated intradural Rosai-Dorfman disease of the spine: report of two cases. Chinese Neurosurg J 2016;2:1

8 Mehraein Y, Wagner M, Remberger K, et al. Parvovirus B19 detected in Rosai-Dorfman disease in nodal and extranodal manifestations. J Clin Pathol 2006;59(12):1320-1326
9 Kattner KA, Stroink AR, Roth TC, Lee JM. Rosai-Dorfman disease mimicking parasagittal meningioma: case presentation and review of literature. Surg Neurol 2000;53(5):452-457, discussion 457

10 Hargett C, Bassett T. Atypical presentation of sinus histiocytosis with massive lymphadenopathy as an epidural spinal cord tumor: a case presentation and literature review. J Spinal Disord Tech 2005;18(2):193-196

11 Roy C, Saha A, Roy S, Ghosh A. Extranodal Rosai-Dorfman disease presenting as spinal extradural lesion: a case report with a review of the literature. J Cancer Res Ther 2012;8(4):647-649

12 Sciacca S, Barkas K, Heptinstall L, McNamara C, Shetty R. Rosai-Dorfman disease with spinal cord compression: a diagnostic challenge. Eur Spine J 2015;24(Suppl 4):S529-S535

13 Rosai J, Dorfman RF. Sinus histiocytosis with massive lymphadenopathy: a pseudolymphomatous benign disorder. Analysis of 34 cases. Cancer 1972;30(5):1174-1188

14 Dran G, Rasendrarijao D, Vandenbos F, Paquis P. Rosai-Dorfman disease causing spinal cord compression: case report. Neurosurgery 2008;62(4):E977-E978, discussion E978

15 Zhu F, Zhang JT, Xing XW, et al. Rosai-Dorfman disease: a retrospective analysis of 13 cases. Am J Med Sci 2013;345(3):200-210

16 Petzold A, Thom M, Powell M, Plant GT. Relapsing intracranial Rosai-Dorfman disease. J Neurol Neurosurg Psychiatry 2001;71(4):538-541

17 Ma J, Xiao J, Wang L. Extranodal Rosai-Dorfman disease with multilevel lumbar spinal lesions. J Neurosurg Spine 2008;9(1):55-57

18 Konca C, Özkurt ZN, Deger M, Akı Z, Yağcı M. Extranodal multifocal Rosai-Dorfman disease: response to 2-chlorodeoxyadenosine treatment. Int J Hematol 2009;89(1):58-62

19 Seyednejad F, Tubbs RS, Shoja MM, Daghigi MH, Oakes WJ. Presumed recurrence of intracranial Rosai-Dorfman disease as a cervical spine tumor. Acta Neurochir (Wien) 2007;149(4):425-427 